\title{
HIV-I Replication Requires an Intact Integrase Reading Frame
}

H. D. Buchow, E. Tschachler, R. C. Gallo, and M. Reitz Jr.

Human immunodeficiency virus type I (HIV-I), is a human retrovirus that is the causative agent of acquired immune deficiency syndrome (AIDS). Retroviruses replicate through a DNA intermediate. After initial synthesis of unintegrated DNA the insertion of that DNA into the host cell genome is thought to be a required step in the retroviral life cycle [1].

A hallmark of HIV-I infection, however, is the persistent appearance of large amounts of unintegrated DNA. Integrated DNA is often difficult to detect, and is readily detectable only after extensive passage of infected cell lines. Consequently, it is not clear that integration is an obligatory part of the HIV-I life cycle. To test the need for integration in HIV-I replication and expression, we have introduced point mutations including a stop codon into the $\mathrm{COOH}$ terminal part of the POL gene containing the integrase coding region and substituted this region for the analogous region of the biologically active molecular clone of HIV-I, pHXB2D [2]. Transfection of cos-I cells with the INT mutant resulted in transient expression of biologically active virus. However, infection of $\mathrm{H} 9$ cells with the mutant virus yielded neither detectable persistent media reverse transcriptase activity nor viral GAG antigen. These results suggest that integration of proviral DNA is a necessary part of the productive infection of T-cells.

After infection, retroviral DNA synthesis results in three types of unintegrated DNA: the linear double-stranded

National Institutes of Health, National Cancer Institute, Bethesda, USA
DNA in the cytoplasm and two circular species with a single LTR or two tandem LTRs, respectively, in the nucleus (Fig. 1), the former being most likely the immediate precursor of the integrated DNA [3]. The process of integration requires an integrase activity that is encoded by the $3^{\prime}$-terminal region of the POL gene [4]. To evaluate the need for integration in HIV-I replication and expression, we introduced point mutations into this region. Using the M13 oligonucleotide-directed mutagenesis system [5], we changed two bases in a $1.1 \mathrm{~kb}$ EcoRI/ EcoRI fragment of the biologically active molecular clone pHXB2D (Fig. 2), which had been cloned into the bacteriophage M13 mp18. Synthesized mismatched oligonucleotides containing a premature stop codon and an additional recognition site for the enzyme BamHI were used to prime second-strand mutagenesis with M13 single-stranded DNA. Purified circular double-stranded DNA was isolated using the alkaline lysis method [6]. The replicative forms of M13 mutant clones were grown and the modified EcoRI/ EcoRI inserts were cloned back into pHXB2D replacing the wild type EcoRI/ EcoRI fragment.

The resultant $\mathrm{pHXB} 2 \mathrm{D} / \mathrm{INT}$ mutant clones were identified and confirmed by restriction enzyme digested with either KpnI, which yielded identical fragments for both the pHXB2D wild type and the INT mutant, and with BamHI, which gave an additional $3.7 \mathrm{~kb}$ fragment only with the INT mutant. Plasmids with the INT mutation were grown in HB101 cells and transfected into cos-I cells. After 62 to $72 \mathrm{~h}$ the virus was introduced into $\mathrm{H} 9$ cells by either cocultivation with the 


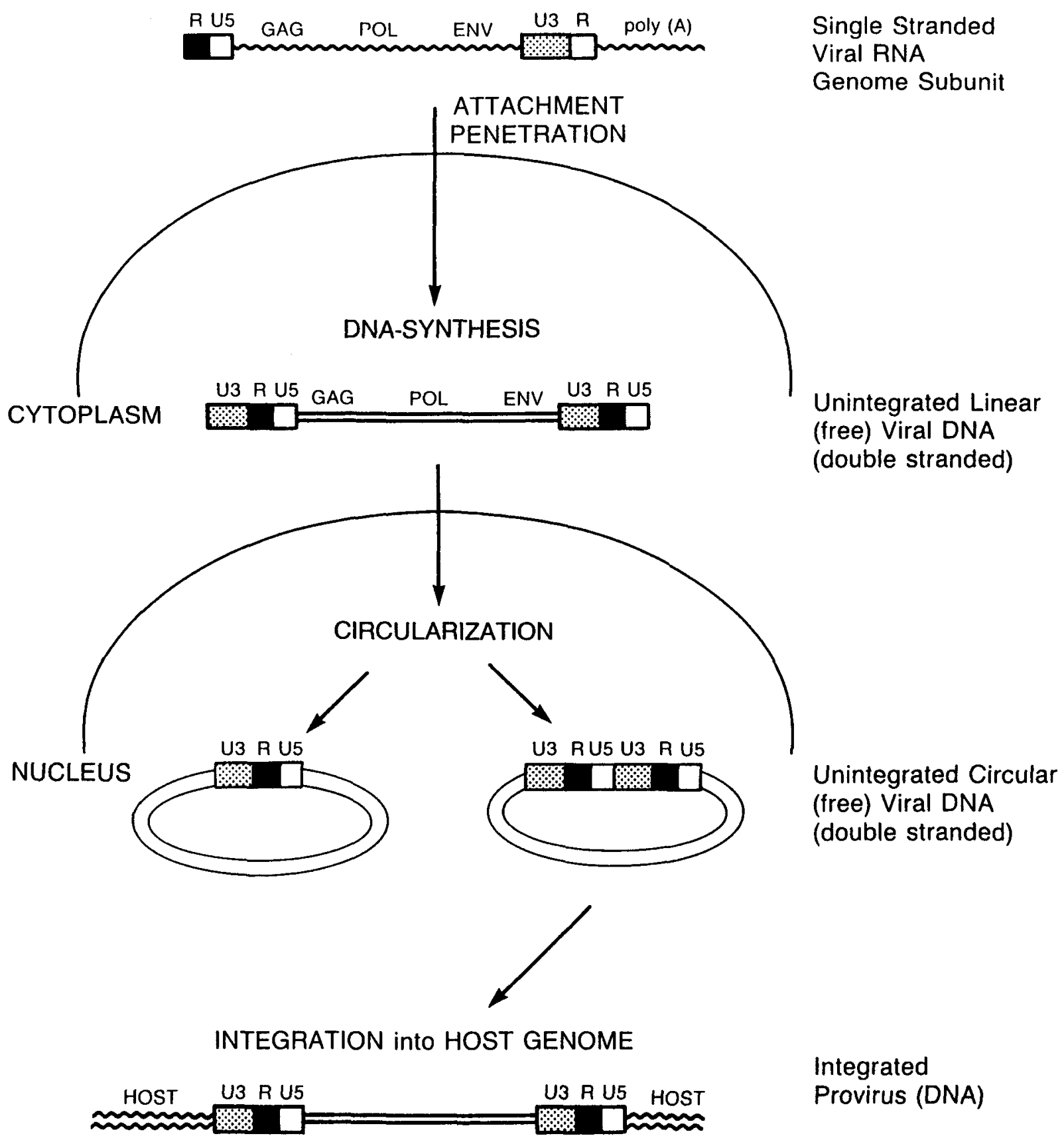

Fig. 1. Integration of retrovirus DNA

transfected cells or by cell-free infection with culture fluids of the same cos-I cell cultures. Transfection of the mutant into cos-I cells yielded media reverse transcriptase (RT) activity, although at a threefold lower level than the wild type HIV-I plasmid.

In radioimmunoprecipitation assays with ${ }^{125}$ I-protein A, lysates of the transfected cos-I cells were immunoprecipitated with antisera from different HIV-I infected patients. For transfections with both the HIV-I wild type and the mutant virus, the assay resulted in the appearance of at least three bands in the gel at 24, 39 and $55 \mathrm{kDa}$, that were not found with controls with mock transfected cells or with sera of HIV-I negative patients, most likely representing the GAG protein $\mathrm{p} 24$, the GAG precursor protein $\mathrm{p} 39$, and the reverse transcriptase $\mathrm{p} 55$.

To evaluate the expression of viral GAG antigen by $\mathrm{H} 9$ cells that were infected by cocultivation with transfected cos-I cells or cell-free with the appropriate culture fluid as described above, we 


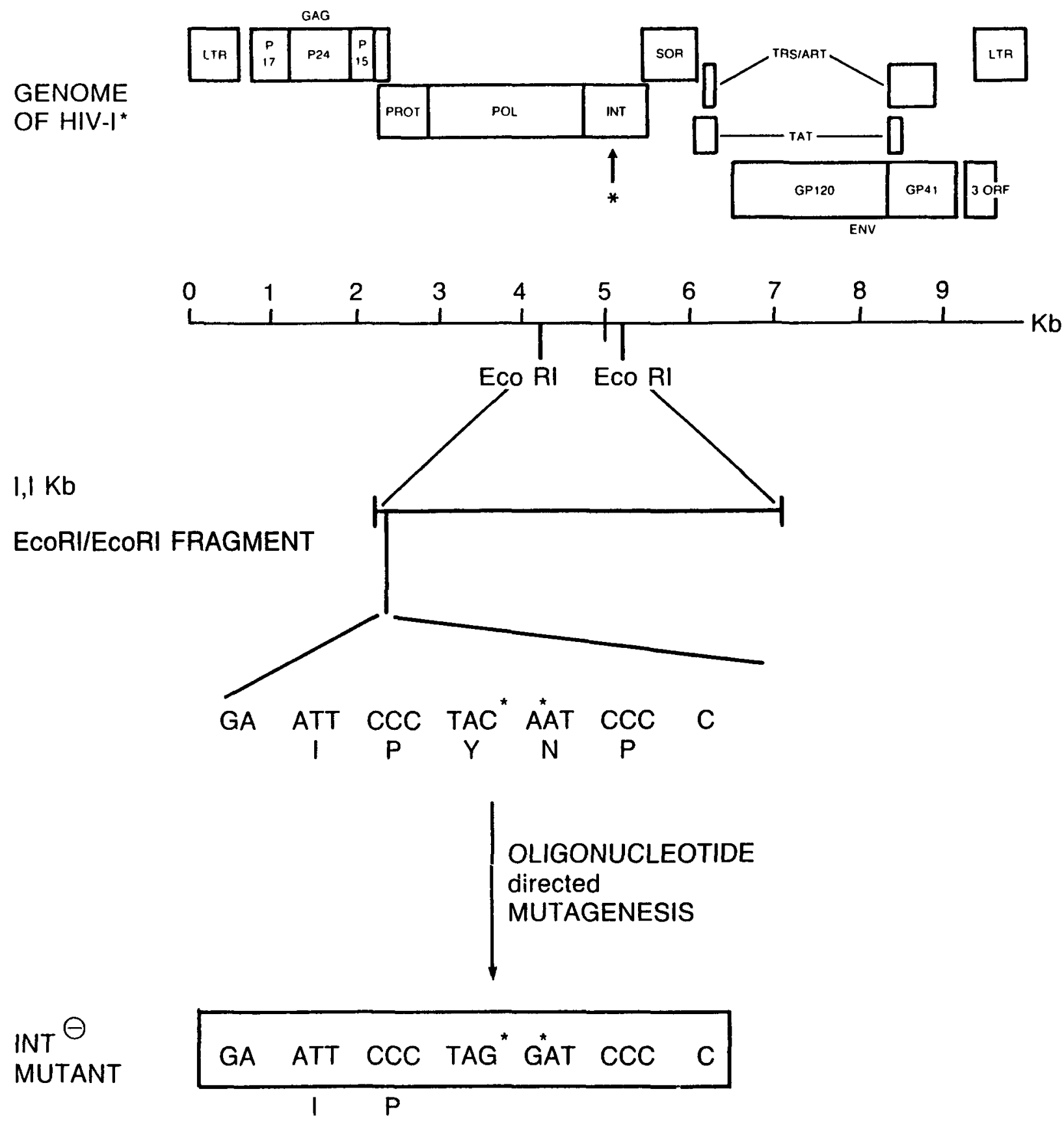

Fig. 2. Construction of INT mutants of HIV-I

used an immunofluorescence assay with anti-p24-antibodies. Both modes of infection resulted in $90 \%$ to $100 \%$ p 24 expression of $\mathrm{H} 9$ cells infected with HIV-I wild type, while neither cell-free infection nor infection by cocultivation with the HIV-I/INT mutant showed p24 expression until day 30 . Assays for RT activity of the same $\mathrm{H} 9$ cultures infected with the HIV-I wild type virus resulted in 78000 and $35000 \mathrm{cpm} / \mathrm{ml}$ culture fluid 2 weeks after infection by cocultivation with transfected cos-I cells or with the appro- priate cell-free supernatants, respectively. In contrast to this, no RT activity was detected in culture fluids of $\mathrm{H} 9$ cells that were cocultivated with or cultured in cell-free supernatant of HIV-I/INT-mutant transfected cos-I cells for various time periods up to 4 weeks.

These results suggest that integration of proviral DNA is a necessary part of the productive infection of $T$ cells. Further studies on expression of viral proteins and mRNA by this mutant are in progress. The requirement for integra- 
tion for reproduction after infection of non-dividing susceptible cells such as monocyte/macrophages remains to be determined.

Acknowledgement. H. D. Buchow was supported by a grant from the $\mathrm{H}$. and L. Schilling Foundation, F.R.G.

\section{Reference}

1. Varmus H, Swanstrom R (1984) Replication of retroviruses. In. Weiss $R$ et al. (eds) RNA tumor viruses, 2nd ed. Cold Spring Harbor Lab, New York
2. Fisher AG et al. (1985) A molecular clone of HTLV-III with biological activity. Nature 316:262-265

3. Panganiban AT (1985) Retroviral DNA integration. Cell 42:5-6

4. Panganiban A, Temin H (1984) The retrovirus $P O L$ gene encodes a product required for DNA integration: identification of a retrovirus INT locus. Proc Natl Acad Sci USA 81:7885-7889

5. Zoller MJ, Smith M (1984) Oligonucleotide-directed mutagenesis: a simple method using two oligonucleotide primers and a single-stranded DNA template. DNA 3:479-488

6. Maniatis T, Fritsch EF, Sambrook J (1982) Molecular cloning. A laboratory manual. Cold Spring Harbor Lab, New York 